\title{
A periodic and seasonal statistical model for non-negative integer-valued time series with an application to dispensed medications in respiratory diseases
}

\author{
Paulo Roberto Prezotti Filho ${ }^{\mathrm{a}, \mathrm{b}, \mathrm{c}}$, Valderio Anselmo Reisen ${ }^{\mathrm{b}, \mathrm{c}, *}$, Pascal \\ Bondon $^{\mathrm{c}}$, Márton Ispány ${ }^{\mathrm{d}}$, Milena Machado Melo ${ }^{\mathrm{a}}$, Faradiba Sarquis ${ }^{\mathrm{e}}$ \\ ${ }^{a}$ Federal Institute of Espirito Santo, IFES, Brazil \\ ${ }^{b}$ Federal University of Espirito Santo, PPGEA-CT-UFES, Brazil \\ ${ }^{c}$ Université Paris-Saclay, CNRS, CentraleSupélec, Laboratoire des Signaux et Systèmes, \\ 91190, Gif-sur-Yvette, France \\ ${ }^{d}$ Faculty of Informatics, University of Debrecen, Hungary \\ ${ }^{e}$ EMESCAM, Brazil
}

\begin{abstract}
This paper introduces a new class of models for non-negative integer-valued time series with a periodic and seasonal autoregressive structure. Some properties of the model are discussed and the conditional quasi-maximum likelihood method is used to estimate the parameters. The consistency and asymptotic normality of the estimators are established. Their performances are investigated for finite sample sizes and the empirical results indicate that the method gives accurate estimates. The proposed model is applied to analyse the daily number of antibiotic dispensing medication for the treatment of respiratory diseases, registered in a health center of Vitória, Brazil.
\end{abstract}

Keywords: Count time series, periodicity, seasonality, consistency, forecast, air pollution problems.

\footnotetext{
* Corresponding author

Email address: valderio.reisen@ufes.br (Valderio Anselmo Reisen )
} 


\section{Introduction}

The study of medicine dispensing has become an important research topic since it can be very useful for public health actions such as to control and detect epidemic diseases, to promote public health education campaign, to reduce cost, to improve the quality of care, to propose intervention strategies, among others; see, for example, [1]. The papers [2], [3, 4, 5, [6] are some recent publications related to this subject.

The model proposed in this paper is mainly motivated by the analysis of the count time series of the daily number of people who received antibiotics for respiratory diseases treatment from public health care system in the emergency service in the region of Vitória-ES (Brazil). Since the respiratory diseases are strongly correlated to air pollution levels and weather conditions, the correlation structure of the daily number of people who received antibiotics presents, among other phenomena, periodicity and seasonality.

A count time series may be represented by the integer-valued autoregressive (INAR) class of models, for example, the INAR processes with autoregressive order 1 (INAR(1)), which was initially introduced in 7 and, independently, in [8]. The INAR(1) model has been widely investigated from theoretical and applied point of views. More recently one can cite, for example, 9], 10] and [11. The two former papers presented inferential aspects of INAR(1) process for zero-inflated time series and the latter used this model to fit heavy-tailed count time series.

The INAR(1) model is based on the thinning operator, defined as follows, see [12. Let $Y$ be a $\mathbb{Z}_{+}$-valued random variable (r.v.) and $\alpha \in[0,1]$. The binomial thinning operator $\circ$ is defined as

$$
\alpha \circ Y=\sum_{i=1}^{Y} U_{i}(\alpha)
$$

where $\left\{U_{i}(\alpha)\right\}_{i \in \mathbb{N}}$ is a sequence of independent identically distributed (i.i.d.) r.v.'s which are Bernoulli distributed with parameter $\alpha$. It is assumed that the sequence $\left\{U_{i}(\alpha)\right\}_{i \in \mathbb{N}}$ is independent of $Y$. Note that the empty sum is set to 0 if 
$Y=0$. The sequence $\left\{U_{i}(\alpha)\right\}_{i \in \mathbb{N}}$ is called a counting process. The probability of success in the thinning is $\mathrm{P}\left(U_{i}(\alpha)=1\right)=\alpha$ and, conditionally on $Y, \alpha \circ Y$ is distributed according to the binomial distribution $\operatorname{Bin}(Y, \alpha)$. For more details on thinning based count time series models see, e.g., 13 in the univariate and [14] in the multivariate case, respectively.

An extension of the INAR(1) model that takes into account the $p$-th order autoregressive structure is the $\operatorname{INAR}(p)$, introduced in [15] and, independently in [16]. The authors in [15] introduced a model for count time series that has a correlation structure similar to the correlation structure of a conventional $\operatorname{ARMA}(p, p-1)$ model for continuous data. Du and Li [16] suggested a model based on a process with a correlation structure identical to the correlation structure of a standard $\operatorname{AR}(p)$ model.

In [16, despite its flexibility in dealing with higher order autoregressive processes, the $\operatorname{INAR}(p)$ model does not account for the periodic phenomenon, which is a quite common time series characteristic in many areas of application, especially, in the air quality and health area. Stochastic processes with periodically varying mean, variance and covariance were introduced in [17 and are usually called periodically correlated (PC) processes. The occurrence of $\mathrm{PC}$ processes in time series is corroborated by real applications in many practical situations, see, e.g., [18]. Basawa and Lund [19] studied the asymptotic properties of parameter estimates for specific periodic autoregressive moving-average (PARMA) models among others. Recently, robust estimation methods for periodic autoregressive (PAR) models were applied to air pollution data in [20] and [21].

Even though there are many studies in the literature that focus on PC processes, most of them are dedicated to the analysis and applications for discrete time processes with continuous marginal distributions, like the PARMA model. The analysis of PC count time series was discussed in [22, [23], 24], 25], 26] and 27]. Monteiro et al. [22] introduced the periodic $\operatorname{INAR}(1)(\operatorname{PINAR}(1))$ model and addressed some statistical properties of the parameter estimation together with some finite sample size investigations. However, the paper did not explore the model in a practical problem. Sadoun and Bentarzi [23] provided 
efficient estimation methods of a PINAR(1) model. Morina et al. 24] presented an $\operatorname{INAR}(2)$ model with seasonal behavior in the immigration to analyze the number of hospital emergency service arrivals caused by diseases. Bourguigon et al. 25] introduced a seasonal INAR model of order 1. In [26, the model discussed in [25] was generalized in the sense that the autoregressive parameters vary by seasons and the immigration processes are allowed to be intra-seasonally dependent. Liu et al. 27] proposed a generalization of the $r$ states random environment INAR(1) model, introduced in [28, to predict time series of counts with small values and notable fluctuations.

The interest of using INAR processes in count time series and their extensions, from theoretical and applied point of views, is still a growing subject in the literature with a large field of applications. For example, recently, Bentarzi and Aries [29] introduced the periodic integer-valued $\operatorname{ARMA}(p, q)$ model, denoted by $\operatorname{PINARMA}_{S}(p, q)$, and established the consistency and the asymptotic normality of the quasi maximum likelihood estimator. This model is also considered here, in the application section, for comparison purpose.

This paper is dedicated to introduce a new class of models called $\operatorname{PINAR}\left(1,1_{S}\right)$ extending the ones in [24] and [22], in order to deal with $\mathbb{Z}_{+}$-valued time series with a periodic and seasonal autoregressive structure. The theoretical properties of the proposed model such as existence, uniqueness and periodic stationarity conditions are established as well as the asymptotic properties of the conditional quasi-maximum likelihood (CQML) estimator. The model is tested with the real data set of dispensed medications and is shown to outperform the other competitive existing models.

The paper is organized as follows. Section 2 introduces the model and its properties. The estimation method and the forecast are shown in Section 3. Section 4 presents finite sample size investigation. Section 5 deals with a real application and conclusions are presented in Section 6. The proofs appear in the Appendix. 


\section{The PINAR $\left(1,1_{S}\right)$ model}

In the following, $\left\{Y_{t}\right\}_{t \in \mathbb{Z}}$ is a stochastic count process with periodic characteristics of period $S, S \in \mathbb{N}$, defined on a probability space $(\Omega, \mathcal{A}, \mathrm{P})$, and depending on an unknown parameter vector $\boldsymbol{\vartheta}$. We denote by $\mathcal{F}_{t}$ the sigma field generated by the r.v.'s $Y_{l}$ for $l \leq t$, and by $\mathrm{E}(\cdot)$ and $\mathrm{E}(\cdot \mid \cdot)$, the expectation and conditional expectation, respectively, under the probability measure $\mathrm{P}$ and the true parameter vector $\boldsymbol{\vartheta}_{0}$. The time index $t$ may be written, by Euclidean division, as $t=k S+\nu$, where $\nu=1, \ldots, S$ and $k \in \mathbb{Z}$. For example, in the case of daily data studied here, $S=7, \nu$ and $k$ represent the day of the week and the week, respectively.

Definition 1. $\left\{Y_{t}\right\}_{t \in \mathbb{Z}}$ is said to be a periodic $\mathbb{Z}_{+}$-valued process with period $S \in \mathbb{N}$ and autoregressive orders $\left(1,1_{S}\right)$, and is denoted by $\operatorname{PINAR}\left(1,1_{S}\right)$, if it satisfies the following stochastic recursion

$$
Y_{k S+\nu}=\alpha_{\nu} \circ Y_{k S+\nu-1}+\beta_{\nu} \circ Y_{k S+\nu-S}+\varepsilon_{k S+\nu}
$$

where $k \in \mathbb{Z}, \nu=1, \ldots, S, \alpha_{\nu}, \beta_{\nu} \in(0,1)$ are the autoregressive coefficients during the season $\nu$. The immigration process $\left\{\varepsilon_{t}\right\}_{t \in \mathbb{Z}}$ is a sequence of independent $\mathbb{Z}_{+}$-valued r.v.'s such that for each $\nu=1, \ldots, S$, the r.v.'s $\left\{\varepsilon_{k S+\nu}\right\}_{k \in \mathbb{Z}}$ are identically distributed with mean $\mathrm{E}\left(\varepsilon_{k S+\nu}\right)=\lambda_{\nu}$, and finite variance $\operatorname{Var}\left(\varepsilon_{k S+\nu}\right)=$ $\sigma_{\nu}^{2}>0$. In addition, it is assumed that $\varepsilon_{t}$ is independent of $Y_{t-1}, \alpha_{\nu} \circ Y_{t-1}$, $Y_{t-S}$ and $\beta_{\nu} \circ Y_{t-S}$ and all counting processes are mutually independent.

In this paper, we assume that $\left\{Y_{t}\right\}_{t \in \mathbb{Z}}$ satisfies 22 where $\left\{\varepsilon_{t}\right\}_{t \in \mathbb{Z}}$ is Poisson distributed. Then $\sigma_{\nu}^{2}=\lambda_{\nu}$ and $\left\{Y_{t}\right\}_{t \in \mathbb{Z}}$ depends on the unknown $3 S$ dimensional parameter vector $\boldsymbol{\vartheta}=\left(\alpha_{1}, \beta_{1}, \lambda_{1}, \ldots, \alpha_{S}, \beta_{S}, \lambda_{S}\right)^{\top}$, where "T" means transpose. As can be seen, for each seasonal period $\nu, Y_{t}$ has three random components; the elements of the immediate past $Y_{t-1}$ with survival probability $\alpha_{\nu}$, the elements of the seasonal past $Y_{t-S}$ with survival probability $\beta_{\nu}$ and the immigration $\varepsilon_{t}$ which entered in the system in the interval $(t-1, t]$. Moreover, the autoregressive parameters $\alpha_{\nu}, \beta_{\nu}$ and the immigration mean $\lambda_{\nu}$ change periodically according to the seasonal period $S$. Observe that 
the above model extends the models introduced in [24] and [22]. For example, the autoregressive coefficients in 24] are fixed, whereas they vary periodically in the PINAR $\left(1,1_{S}\right)$ model. The existence and uniqueness properties of the PINAR $\left(1,1_{S}\right)$ process can be obtained analogously as the standard PC ARMA process introduced in [19, and using the properties of the multivariate integervalued autoregressive process $(\operatorname{MGINAR}(p))$ introduced in [14]. Following the same lines of the matrix representation of the PARMA process in [19] and the $\operatorname{MGINAR}(p)$ model, some properties of model (2) are now discussed.

Let $A=\left[a_{i, j}\right]$ and $B=\left[b_{i, j}\right]$ be the $S \times S$ matrices with non-negative elements defined by

$$
a_{i j}=\left\{\begin{array}{ll}
\alpha_{i} & \text { if } i=j+1, \\
0 & \text { otherwise, }
\end{array} \quad \text { and } \quad b_{i j}= \begin{cases}\beta_{i} & \text { if } i=j, \\
\alpha_{1} & \text { if } i=1, j=S, \\
0 & \text { otherwise }\end{cases}\right.
$$

$A$ is strictly lower triangular and $B$ is upper triangular. Let $\boldsymbol{Y}_{k}=\left(Y_{k S+1}, \ldots\right.$, $\left.Y_{k S+S}\right)^{\top}, \varepsilon_{k}=\left(\varepsilon_{k S+1}, \ldots, \varepsilon_{k S+S}\right)^{\top}$, and $A \circ=\left[a_{i, j} \circ\right], B \circ=\left[b_{i, j} \circ\right]$ be matricial binomial thinning operators [14, Definition 2.1]. The action of $A \circ$ on $\boldsymbol{Y}_{k}$ is defined by

$$
A \circ \boldsymbol{Y}_{k}=A \circ\left(\begin{array}{c}
Y_{k S+1} \\
\vdots \\
Y_{k S+S}
\end{array}\right)=\left(\begin{array}{c}
\sum_{j=1}^{S} a_{1, j} \circ Y_{k S+j} \\
\vdots \\
\sum_{j=1}^{S} a_{S, j} \circ Y_{k S+j}
\end{array}\right)
$$

Using (2), the following stochastic equation holds

$$
\boldsymbol{Y}_{k}=A \circ \boldsymbol{Y}_{k}+B \circ \boldsymbol{Y}_{k-1}+\varepsilon_{k},
$$

where $A$ and $B$ are defined by (3). All the counting sequences involved in the thinning operators $a_{i, j}$ 的 $b_{i, j}$, for $1 \leq i, j \leq S$, are mutually independent and are independent of the sequence $\left\{\varepsilon_{t}\right\}_{t \in \mathbb{Z}}$. For a strictly lower triangular nonnegative matrix $M$ and a $\mathbb{Z}_{+}$-valued random vector $\boldsymbol{Y}$, define $(I-M) \circ \boldsymbol{Y}:=$ $\boldsymbol{Y}-M \circ \boldsymbol{Y}$. Then, (4) can be written as

$$
(I-A) \circ \boldsymbol{Y}_{k}=B \circ \boldsymbol{Y}_{k-1}+\varepsilon_{k},
$$


which can be considered as the state-space representation of the $\operatorname{PINAR}\left(1,1_{S}\right)$ process with analogy to the state-space representation of a PARMA process see, e.g., [19, Equation (2.3)].

Now, suppose that $\left\{\boldsymbol{Y}_{k}\right\}_{k \in \mathbb{Z}}$ has a constant mean vector $\boldsymbol{\mu}$ for all $k \in \mathbb{Z}$. Using [14, Lemma 2.1] and taking the expectation of (4), we get that

$$
\boldsymbol{\mu}=(A+B) \boldsymbol{\mu}+\boldsymbol{\lambda},
$$

which can be written

$$
(I-(A+B)) \boldsymbol{\mu}=\boldsymbol{\lambda},
$$

or equivalently,

$$
(I-A) \boldsymbol{\mu}=B \boldsymbol{\mu}+\boldsymbol{\lambda} .
$$

$I-A$ is a lower triangular non-singular matrix whose inverse $A^{*}=\left[a_{i j}^{*}\right]$, is given by

$$
a_{i j}^{*}= \begin{cases}1 & \text { if } i=j, \\ \prod_{k=j+1}^{i} \alpha_{k} & \text { if } i>j, \\ 0 & \text { if } i<j .\end{cases}
$$

Then, (2) is equivalent to

$$
\boldsymbol{\mu}=A^{*} B \boldsymbol{\mu}+A^{*} \boldsymbol{\lambda}
$$

that is,

$$
\left(I-A^{*} B\right) \boldsymbol{\mu}=A^{*} \boldsymbol{\lambda} .
$$

The existence of a non-negative solution $\boldsymbol{\mu}$ to (5) and (6) depends on the spectral properties of the matrices $A+B$ and $A^{*} B$, respectively. One can see that $A+B$ is a Perron-Frobenius matrix, i.e., non-negative and irreducible, see [30, Definition 6.2.22]. Let $\rho(M)$ denote the spectral radius of a matrix $M$ of dimension $S \times S$, which is the maximum eigenvalue in modulus of $M$. By [31, Theorem 2.1], a necessary and sufficient condition for a solution $\boldsymbol{\mu}$ $(\boldsymbol{\mu} \geq \mathbf{0}, \neq \mathbf{0})$, where $\mathbf{0}$ is a $S$-dimensional vector of zeros, to 5 to exist for any $\boldsymbol{\lambda}(\boldsymbol{\lambda} \geq \mathbf{0}, \neq \mathbf{0})$ is that $\rho(A+B)<1$. Note that, since $S \geq 2$, from the Perron-Frobenius Theorem [30, Theorem 8.4.4], $\rho(A+B)>0$ and $\rho(A+B)$ is 
an algebraically simple eigenvalue of $A+B$. The condition $\rho(A+B)<1$ implies that $\sum_{k=1}^{\infty}(A+B)^{k}$ converges to $(I-(A+B))^{-1}$, see [30, Theorem 5.6.15 and Corollary 5.6.14]. Thus, condition $\rho(A+B)<1$ assures the invertibility of $I-(A+B)$ and the non-negativity of its inverse, see [32, page 100]. In addition, $\boldsymbol{\mu}=(I-(A+B))^{-1} \boldsymbol{\lambda}$ is the unique solution to (5). Similarly, $\rho\left(A^{*} B\right)<1$ assures the invertibility of $I-A^{*} B$ and the non-negativity of its inverse which is given by the convergent infinite series $\sum_{k=1}^{\infty}\left(A^{*} B\right)^{k}$. Moreover, $\boldsymbol{\mu}^{*}=\left(I-A^{*} B\right)^{-1} A^{*} \boldsymbol{\lambda}$ is the unique solution to $(6)$. One can prove that $\boldsymbol{\mu}=\boldsymbol{\mu}^{*}$ and $\rho(A+B)<1$ is equivalent to $\rho\left(A^{*} B\right)<1$.

Assumption 1. The matrices $A$ and $B$ satisfy $\rho(A+B)<1$.

Remark 1. Note that $\rho(A+B)<1$ is equivalent to : (a) the roots of the determinant equation $\operatorname{det}(z I-(A+B))=0, z \in \mathbb{C}$, are less than 1 in modulus, and, (b) the roots of the characteristic polynomial $P(z)=\prod_{j=1}^{S}\left(z-\beta_{j}\right)-$ $\prod_{j=1}^{S} \alpha_{j}, z \in \mathbb{C}$, lie inside the complex unit circle.

The following lemma can be proved following the same lines as [14, Section 3].

Lemma 1. Under Assumption 1, there exist a unique $\mathbb{Z}_{+}$-valued strictly periodically stationary ergodic process $\left\{Y_{t}\right\}_{t \in \mathbb{Z}}$ satisfying (2) and such that $\varepsilon_{t}$ is independent of $Y_{u}, t>u$.

Example 1. Consider the case when $\beta_{j}=0$ for all $j=1, \ldots, S$. Then the $\operatorname{PINAR}\left(1,1_{S}\right)$ model reduces to the PINAR $(1)_{S}$ model introduced in 22. The characteristic polynomial of this model simplifies to $P(z)=z^{S}-\prod_{j=1}^{S} \alpha_{j}$ and the condition $\rho(A+B)<1$ is equivalent to $\prod_{j=1}^{S} \alpha_{j}<1$. Note that $\prod_{j=1}^{S} \alpha_{j}$ is the spectral radius of the matrix $A$ defined in [22, page 1531].

Example 2. Consider the case $S=2$, i.e., the $\operatorname{PINAR}\left(1,1_{2}\right)$ model. Then

$$
A=\left[\begin{array}{cc}
0 & 0 \\
\alpha_{2} & 0
\end{array}\right], \quad A^{*}=\left[\begin{array}{cc}
1 & 0 \\
\alpha_{2} & 1
\end{array}\right], \quad B=\left[\begin{array}{cc}
\beta_{1} & \alpha_{1} \\
0 & \beta_{2}
\end{array}\right] \text {. }
$$

The characteristic polynomial is given by $P(z)=\left(z-\beta_{1}\right)\left(z-\beta_{2}\right)-\alpha_{1} \alpha_{2}$. By solving the characteristic equation, it can be seen that $\beta_{1}+\beta_{2}-\beta_{1} \beta_{2}+\alpha_{1} \alpha_{2}<1$ 
is a necessary and sufficient stationarity condition. Note that this condition can be rewritten as $\alpha_{1} \alpha_{2}<\left(1-\beta_{1}\right)\left(1-\beta_{2}\right)$.

Proposition 1. Let $\left\{Y_{t}\right\}_{t \in \mathbb{Z}}$ be the periodic $\mathbb{Z}_{+}$-valued process defined by (2). Then, the periodic autocovariance functions $\gamma_{i}(h)=\operatorname{Cov}\left(Y_{t}, Y_{t-h}\right), t \equiv(i$ $\bmod S), i=1, \ldots, S$, satisfy the resursion

$$
\gamma_{i}(h)=\alpha_{i} \gamma_{i-1}(h-1)+\beta_{i} \gamma_{i}(h-S),
$$

where $\gamma_{0}=\gamma_{S}$ in case of $i=1$.

The marginal distribution of $\left\{Y_{t}\right\}_{t \in \mathbb{Z}}$ satisfies

$$
P\left(Y_{k S+\nu}=m\right)=\sum_{b_{1}, b_{2}=0}^{\infty} p_{\nu}\left(m \mid b_{1}, b_{2}\right) P\left(Y_{k S+\nu-1}=b_{1}, Y_{k S+\nu-S}=b_{2}\right),
$$

where $m \in \mathbb{Z}_{+}$and $p_{\nu}\left(m \mid b_{1}, b_{2}\right)=P\left(Y_{t}=m \mid Y_{t-1}=b_{1}, Y_{t-S}=b_{2}\right)$ for each $\nu=1, \ldots, S$. When $S=1,22)$ defines the standard INAR(1) model and $\left\{Y_{t}\right\}_{t \in \mathbb{Z}}$ follows a Poisson distribution when $\left\{\varepsilon_{t}\right\}_{t \in \mathbb{Z}}$ is Poisson distributed. When $S>1$, it can be shown that the unconditional mean and variance of $Y_{t}$ are not equal in general, so that the marginal stationary distribution of $Y_{t}$ is no longer Poisson even though the innovations are. However, an approximation to a Poisson distribution can be achieved if $\alpha_{\nu} \cdot \beta_{\nu} \approx 0$ and $Y_{t}$ becomes large, as an application of the law of small numbers (the law of large numbers for the case of small expectation), see [33, 34].

Proposition 2. Given the starting values $Y_{1}, \ldots, Y_{S}$, the conditional joint probability is given by

$$
\begin{array}{r}
P\left(Y_{T}=y_{T}, \ldots, Y_{S+1}=y_{S+1} \mid Y_{S}=y_{S}, \ldots, Y_{1}=y_{1}\right)= \\
\prod_{\nu=1}^{S} \prod_{k=1}^{n-1} p_{\nu}\left(y_{k S+\nu} \mid y_{k S+\nu-1}, y_{k S+\nu-S}\right) .
\end{array}
$$

Since $\left\{\varepsilon_{t}\right\}_{t \in \mathbb{Z}}$ is Poisson distributed, the conditional probability $p_{\nu}(\cdot \mid \cdot, \cdot)$ in 
(7) is given by

$$
\begin{gathered}
p_{\nu}\left(y_{t} \mid y_{t-1}, y_{t-s}\right)=\left[\operatorname{Bin}\left(y_{t-1}, \alpha_{\nu}\right) * \operatorname{Bin}\left(y_{t-s}, \beta_{\nu}\right) * \operatorname{Poi}\left(\lambda_{\nu}\right)\right]\left(y_{t}\right)= \\
\sum_{\left(c_{1}, c_{2}\right) \in \mathcal{J}}\left(\begin{array}{c}
y_{t-1} \\
c_{1}
\end{array}\right) \alpha_{\nu}^{c_{1}}\left(1-\alpha_{\nu}\right)^{y_{t-1}-c_{1}}\left(\begin{array}{c}
y_{t-s} \\
c_{2}
\end{array}\right) \beta_{\nu}^{c_{2}}\left(1-\beta_{\nu}\right)^{y_{t-s}-c_{2}} \\
\frac{\lambda_{\nu}^{y_{t}-c_{1}-c_{2}}}{\left(y_{t}-c_{2}-c_{1}\right) !} \mathrm{e}^{-\lambda_{\nu}}
\end{gathered}
$$

where $*$ denotes the convolution, and the index set $\mathcal{J}$ is defined by $\mathcal{J}=$ $\left\{\left(c_{1}, c_{2}\right) \in \mathbb{Z}_{+}^{2} \mid c_{1} \leq y_{t-1}, c_{2} \leq y_{t-s}, c_{1}+c_{2} \leq y_{t}\right\}$. Note that the definition of $\mathcal{J}$ depends on the values $y_{t}, y_{t-1}, y_{t-s}$.

\section{Estimation and Forecasting}

\subsection{The CQML estimator}

Let $\boldsymbol{\vartheta}=\left(\vartheta_{1}^{\top}, \ldots, \vartheta_{S}^{\top}\right)^{\top}$ be the $3 S$-dimensional unknown parameter vector of the PINAR $\left(1,1_{S}\right)$ model, where $\vartheta_{\nu}=\left(\alpha_{\nu}, \beta_{\nu}, \lambda_{\nu}\right)^{\top}$ and $\vartheta$ lies in the open set $\Theta=\left((0,1)^{2} \times(0, \infty)\right)^{S}$, which contains the true unknown parameter vector, denoted by $\boldsymbol{\vartheta}_{0}=\left(\left(\vartheta_{1}^{0}\right)^{\top}, \ldots,\left(\vartheta_{S}^{0}\right)^{\top}\right)^{\top}$. Let $Y_{1}, \ldots, Y_{T}$ be a sample of size $T=$ $n S$ from the PINAR $\left(1,1_{S}\right)$ process. Conditioned on the first $S$ observations, the CQML estimator suggested here is based on the likelihood type penalty function given in [35, Equation 3.2.61]. This function is

$$
L_{n}(\boldsymbol{\vartheta})=\sum_{k=1}^{n-1} \sum_{\nu=1}^{S}\left[\log \left\{f_{k, \nu}\left(\vartheta_{\nu}\right)\right\}+\left(Y_{k S+\nu}-m_{k, \nu}\left(\vartheta_{\nu}\right)\right)^{2} f_{k, \nu}^{-1}\left(\vartheta_{\nu}\right)\right],
$$

where

$$
m_{k, \nu}\left(\vartheta_{\nu}\right)=\mathrm{E}\left(Y_{k S+\nu} \mid \mathcal{F}_{k S+\nu-1}\right)=\alpha_{\nu} Y_{k S+\nu-1}+\beta_{\nu} Y_{k S+\nu-S}+\lambda_{\nu}
$$

is the conditional mean and

$$
\begin{aligned}
f_{k, \nu}\left(\vartheta_{\nu}\right) & =\mathrm{E}\left[\left\{Y_{k S+\nu}-m_{k, \nu}\left(\vartheta_{\nu}\right)\right\}^{2} \mid \mathcal{F}_{k S+\nu-1}\right] \\
& =\alpha_{\nu}\left(1-\alpha_{\nu}\right) Y_{k S+\nu-1}+\beta_{\nu}\left(1-\beta_{\nu}\right) Y_{k S+\nu-S}+\lambda_{\nu}
\end{aligned}
$$

is the conditional variance. Note that $m_{k, \nu}\left(\vartheta_{\nu}\right)$ and $f_{k, \nu}\left(\vartheta_{\nu}\right)$ are almost surely three times continuously differentiable in the open set $\Theta$ containing the true parameter value $\boldsymbol{\vartheta}_{0}$. This is one condition stated in [35, Theorem 3.2.26] to obtain 
the asymptotic property of the CQLM estimator established in Theorem 1. Let

$$
\begin{aligned}
l_{n, \nu}\left(\vartheta_{\nu}\right) & =\sum_{k=1}^{n-1}\left[\log \left\{f_{k, \nu}\left(\vartheta_{\nu}\right)\right\}+\left(Y_{k S+\nu}-m_{k, \nu}\left(\vartheta_{\nu}\right)\right)^{2} f_{k, \nu}\left(\vartheta_{\nu}\right)^{-1}\right] \\
& =\sum_{k=1}^{n-1} \phi_{k, \nu}\left(\vartheta_{\nu}\right) .
\end{aligned}
$$

Then $L_{n}(\boldsymbol{\vartheta})=\sum_{\nu=1}^{S} l_{n, \nu}\left(\vartheta_{\nu}\right)$ is minimized to obtain the CQML-estimator $\widehat{\boldsymbol{\vartheta}}_{n}^{\mathrm{CQML}}$ of $\boldsymbol{\vartheta}_{0}$. Observe that the minimization of $L_{n}(\boldsymbol{\vartheta})$ can be done separately by minimizing $l_{n, \nu}\left(\vartheta_{\nu}\right)$ for each season $\nu=1, \ldots, S$, i.e, by solving the equations

$$
\frac{\partial}{\partial \vartheta_{\nu}} l_{n, \nu}\left(\vartheta_{\nu}\right)=0, \quad \nu=1, \ldots, S .
$$

For each $\nu=1, \ldots, S$, we define the matrix $M_{\nu}$ of dimension $3 \times 3$ by

$$
M_{\nu}=U_{\nu}^{-1} V_{\nu} U_{\nu}^{-1}
$$

where

$$
V_{\nu}=\mathrm{E}\left\{\frac{\partial}{\partial \vartheta_{\nu}} \phi_{k, \nu}\left(\vartheta_{\nu}^{0}\right) \frac{\partial}{\partial \vartheta_{\nu}^{\top}} \phi_{k, \nu}\left(\vartheta_{\nu}^{0}\right)\right\}
$$

and

$$
U_{\nu}=\mathrm{E}\left\{\frac{\partial^{2}}{\partial \vartheta_{\nu} \partial \vartheta_{\nu}^{\top}} \phi_{k, \nu}\left(\vartheta_{\nu}^{0}\right)\right\}
$$

The asymptotic covariance matrix $M$ of the CQML estimator of the parameters of the $\operatorname{PINAR}\left(1,1_{S}\right)$ process is given by

$$
M=\operatorname{diag}\left\{M_{1}, \ldots, M_{S}\right\}
$$

Theorem 1. Under assumption 1 , $\left\{Y_{t}\right\}_{t \in \mathbb{Z}}$ defined by (2) is a strictly periodically stationary ergodic process with finite fourth-order moment and the CQML estimator $\widehat{\boldsymbol{\vartheta}}_{n}^{\mathrm{CQML}}$ is asymptotically normal distributed,

$$
n^{1 / 2}\left(\widehat{\boldsymbol{\vartheta}}_{n}^{\mathrm{CQML}}-\boldsymbol{\vartheta}_{0}\right) \stackrel{\mathcal{D}}{\longrightarrow} \mathcal{N}(\mathbf{0}, M)
$$

when $n \rightarrow \infty$, where $M$ is the matrix of dimension $3 S \times 3 S$ defined by 12 .

Corollary 1. $\widehat{\boldsymbol{\vartheta}}_{n}^{\mathrm{CQML}}$ is a consistent estimator of $\boldsymbol{\vartheta}_{0}$. 


\subsection{Forecasting}

Let $Y_{1}, \ldots, Y_{T}$ be a sample from a $\operatorname{PINAR}\left(1,1_{S}\right)$ process with $n$ complete periods of size $S$, i.e., $T=n S, n \in \mathbb{N}$. The forecasting method discussed here is an extension of the approach presented in [16, Section 5]. The minimum variance predictor of $Y_{T+1}$, denoted by $\widehat{Y}_{T}(1)$, is given by

$$
\widehat{Y}_{T}(1)=\mathrm{E}\left(Y_{T+1} \mid \mathcal{F}_{T}\right)=\alpha_{1} Y_{T}+\beta_{1} Y_{T-S+1}+\lambda_{1}
$$

For any $h \in \mathbb{N}$ with $h=k S+\nu$, the minimum variance predictor of $Y_{T+h}$ can be calculated as

$$
\widehat{Y}_{T}(h)=\mathrm{E}\left(Y_{T+h} \mid \mathcal{F}_{T}\right)=\mathrm{E}\left(\alpha_{\nu} \circ Y_{T+h-1} \mid \mathcal{F}_{T}\right)+\mathrm{E}\left(\beta_{\nu} \circ Y_{T+h-S} \mid \mathcal{F}_{T}\right)+\lambda_{\nu} .
$$

We have

$$
\mathrm{E}\left(\alpha_{\nu} \circ Y_{T+h-1} \mid \mathcal{F}_{T}\right)=\mathrm{E}\left(\mathrm{E}\left(\alpha_{\nu} \circ Y_{T+h-1} \mid \mathcal{F}_{T+h-1}\right) \mid \mathcal{F}_{T}\right)=\alpha_{\nu} \mathrm{E}\left(Y_{T+h-1} \mid \mathcal{F}_{T}\right)
$$

and

$$
E E\left(Y_{T+h-1} \mid \mathcal{F}_{T}\right)= \begin{cases}\widehat{Y}_{T}(h-1) & \text { if } h>1 \\ Y_{T+h-1} & \text { if } h=1 .\end{cases}
$$

Similarly,

$$
\mathrm{E}\left(\beta_{\nu} \circ Y_{T+h-S} \mid \mathcal{F}_{T}\right)= \begin{cases}\beta_{\nu} \widehat{Y}_{T}(h-S) & \text { if } h>S, \\ \beta_{\nu} Y_{T+h-S} & \text { if } 1 \leq h \leq S .\end{cases}
$$

The forecasting of $Y_{T+h}$ can be obtained recursively as

$$
\widehat{Y}_{T}(h)=\alpha_{\nu} \widehat{Y}_{T}(h-1)+\beta_{\nu} \widehat{Y}_{T}(h-S)+\lambda_{\nu},
$$

where $\widehat{Y}_{T}(h):=Y_{T+h}$ for $h=0,-1, \ldots,-S+1$.

Since $\varepsilon_{\nu} \sim \operatorname{Poi}\left(\lambda_{\nu}\right)$, the probability distribution of $\widehat{Y}_{T}(h)$ conditional to $\mathcal{F}_{T}$, 
can be computed similarly to (8). For example, if $h=2$, it is given by

$$
\begin{gathered}
p_{T}\left(Y_{T+2}=y_{T+2} \mid \mathcal{F}_{T}\right)=p_{T}\left(Y_{T+2}=y_{T+2} \mid Y_{T}=y_{T}, Y_{T-S+2}=y_{T-S+2},\right. \\
\left.Y_{T-S+1}=y_{T-S+1}\right)= \\
\sum_{(m, n, r) \in \mathcal{J}}\left(\begin{array}{c}
y_{T} \\
m
\end{array}\right)\left(\alpha_{2} \alpha_{1}\right)^{m}\left(1-\left(\alpha_{2} \alpha_{1}\right)\right)^{y_{T}-m}\left(\begin{array}{c}
y_{T-S+2} \\
n
\end{array}\right) \beta_{2}^{n}\left(1-\beta_{2}\right)^{y_{T-S+2}-n} \\
\left(\begin{array}{c}
y_{T-S+1} \\
r
\end{array}\right)\left(\alpha_{2} \beta_{1}\right)^{r}\left(1-\alpha_{2} \beta_{1}\right)^{y_{T-S+1}-r} \frac{\lambda_{T+2}^{Y_{T+2}-m-n-r}}{\left(Y_{T+2}-m-n-r\right) !} \mathrm{e}^{-\lambda_{T+2},},
\end{gathered}
$$

where $\lambda_{T+2}:=\alpha_{2} \lambda_{1}+\lambda_{2}$ and the index set $\mathcal{J}$ is defined by $\mathcal{J}=\{(m, n, r) \in$ $\left.\mathbb{Z}_{+}^{3} \mid m \leq y_{T}, n \leq y_{T-S+2}, r \leq y_{T-S+1}, m+n+r \leq y_{T+2}\right\}$. Note that the binomial coefficients $\alpha_{2} \beta_{1}, \beta_{2}$ and $\alpha_{2} \alpha_{1}$ in the above formula form the non-zero entries of the second row of the matrix $A^{*} B$.

\section{Monte Carlo simulations}

The performance of the CQML method is investigated here for a small sample size $T=n S$ generated from a $\operatorname{PINAR}\left(1,1_{S}\right)$ model with $S=4,7$, and $\left\{\varepsilon_{t}\right\}_{t \in \mathbb{Z}}$ is Poisson distributed. The parameters to be estimated and $T$ are displayed in tables. The empirical bias and mean square error (MSE) correspond to the mean of 1000 replications. All simulations were carried out using the $\mathrm{R}$ software.

Tables 1 and 2 display the results for $S=4$ and $S=7$, respectively. In table 2 the true parameter vector is $\boldsymbol{\vartheta}_{0}=(0.31,0.27,4.00,0.35,0.25,3.30,0.29,0.26$, $2.1,0.29,0.39,2.50,0.37,0.27,3.10,0.29,0.22,2.60,0.28,0.33,3.50)^{\top}$. As was expected, in general, the performance of CQML estimator presents estimates quite accurate even for a small sample size. By increasing $n$, the quantities bias and MSE of the estimates decrease, which corroborates the theoretical results in Theorem 1 Since the parameters $\alpha_{\nu}$ and $\beta_{\nu}$, for each $\nu$, correspond to the coefficients of linear relation between $Y_{t}, Y_{t-1}$ and $Y_{t-S}$, respectively, their estimates perform nearly identical, that is, they present similar MSE. On the other hand, although the estimates of $\lambda_{\nu}$ also present accurate results, these are not precisely in terms of MSE as the ones of $\alpha_{\nu}$ and $\beta_{\nu}$. This fact may be mainly due 
to the minimization algorithm to estimate $\lambda_{\nu}$, which is not linearly related to the observations $Y_{t}$. In practice, however, it may not be a big concern. Other parameter values were also considered in the simulation study and, in general, the conclusions were quite similar to those reported here. These results are available upon request.

\section{Real data application}

The time series of counts refers to the daily number of people who got antibiotics for the treatment of respiratory problems from the public health care system in the emergency service of the region of Vitória-ES, Brazil. This real data set was obtained from the network records system welfare of the municipality and corresponds to the period of May 26, 2013 to September 05, 2015, resulting in $T=833$ daily ( $n=119$ weeks) observations. The series displayed in Figure 1 contains persistence oscillation, that is, the mean changes periodically. This is clearly evidenced in the plots of the sample autocorrelation function $(\mathrm{ACF})$, as discussed below.

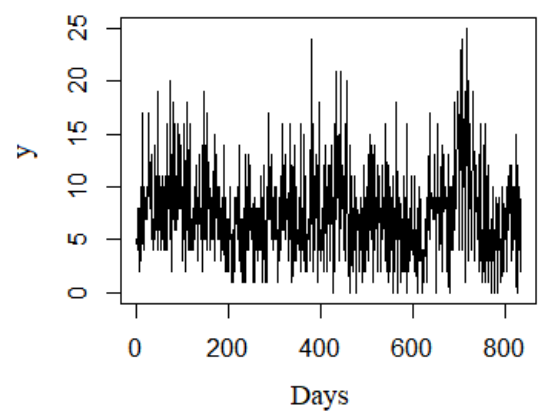

Figure 1: Daily number of people who received antibiotics for the treatment of respiratory problems from the public health care system in the emergency service of the region of VitóriaES.

Figure 2 shows the sample periodic mean and variance of the series over seasons $\nu=1, \ldots, 7$, the sample ACF and the periodogram of the series. The analysis of the sample ACF suggests that this series has seasonal autocorrela- 
Table 1: Results of the simulation to estimate the parameters of the PINAR $\left(1,1_{4}\right)$ model with sample size $T=200,800$ and 2000 values. The true parameter vector is $\boldsymbol{\vartheta}_{0}=$ $(0.10,0.47,4.00,0.42,0.25,3.00,0.23,0.36,2.00,0.39,0.30,1.00)^{\top}$. Inside parenthesis is the MSE of each estimator.

\begin{tabular}{|c|c|c|c|}
\hline & $n=50, T=200$ & $n=200, T=800$ & $n=500, T=2000$ \\
\hline & Bias $_{\mathrm{CQML}}$ & Bias $_{\mathrm{CQML}}$ & Bias $_{\mathrm{CQML}}$ \\
\hline \multirow[t]{2}{*}{$\alpha_{1}$} & 0.025 & -0.002 & -0.004 \\
\hline & $(0.018)$ & $(0.005)$ & $(0.003)$ \\
\hline \multirow[t]{2}{*}{$\alpha_{2}$} & 0.021 & 0.007 & -0.004 \\
\hline & $(0.014)$ & $(0.004)$ & $(0.002)$ \\
\hline \multirow[t]{2}{*}{$\alpha_{3}$} & 0.009 & 0.002 & 0.001 \\
\hline & $(0.013)$ & $(0.003)$ & $(0.001)$ \\
\hline \multirow[t]{2}{*}{$\alpha_{4}$} & 0.004 & 0.006 & 0.000 \\
\hline & $(0.010)$ & $(0.002)$ & $(0.001)$ \\
\hline \multirow[t]{2}{*}{$\beta_{1}$} & -0.028 & -0.008 & 0.002 \\
\hline & $(0.015)$ & $(0.003)$ & $(0.001)$ \\
\hline \multirow[t]{2}{*}{$\beta_{2}$} & -0.024 & -0.007 & -0.005 \\
\hline & $(0.017)$ & $(0.004)$ & $(0.002)$ \\
\hline \multirow[t]{2}{*}{$\beta_{3}$} & -0.035 & -0.006 & -0.003 \\
\hline & $(0.017)$ & $(0.004)$ & $(0.002)$ \\
\hline \multirow[t]{2}{*}{$\beta_{4}$} & -0.011 & -0.005 & -0.003 \\
\hline & $(0.015)$ & $(0.004)$ & $(0.002)$ \\
\hline \multirow[t]{2}{*}{$\lambda_{1}$} & 0.081 & 0.085 & -0.008 \\
\hline & $(1.324)$ & $(0.278)$ & $(0.151)$ \\
\hline \multirow[t]{2}{*}{$\lambda_{2}$} & 0.003 & 0.017 & 0.068 \\
\hline & $(1.427)$ & $(0.342)$ & $(0.157)$ \\
\hline \multirow[t]{2}{*}{$\lambda_{3}$} & 0.11 & 0.005 & 0.015 \\
\hline & $(1.16)$ & $(0.208)$ & $(0.091)$ \\
\hline \multirow[t]{2}{*}{$\lambda_{4}$} & 0.058 & -0.02 & 0.01 \\
\hline & $(0.455)$ & $(0.096)$ & $(0.042)$ \\
\hline
\end{tabular}


Table 2: Results of the simulation to estimate the parameters of the PINAR $\left(1,1_{7}\right)$ model. Inside the parenthesis is the MSE of each estimate.

\begin{tabular}{|c|c|c|c|}
\hline & $n=50, T=350$ & $n=100, T=700$ & $n=200, T=1400$ \\
\hline Pars & $\operatorname{Bias}_{\mathrm{CQML}}$ & Bias $_{\mathrm{CQML}}$ & $\operatorname{Bias}_{\mathrm{CQML}}$ \\
\hline \multirow[t]{2}{*}{$\alpha_{1}$} & 0.017 & 0.005 & 0.003 \\
\hline & $(0.021)$ & $(0.009)$ & $(0.002)$ \\
\hline \multirow[t]{2}{*}{$\alpha_{2}$} & 0.013 & 0.011 & 0.004 \\
\hline & $(0.017)$ & $(0.007)$ & $(0.001)$ \\
\hline \multirow[t]{2}{*}{$\alpha_{3}$} & 0.003 & 0.006 & 0.000 \\
\hline & $(0.011)$ & $(0.006)$ & $(0.001)$ \\
\hline \multirow[t]{2}{*}{$\alpha_{4}$} & 0.005 & 0.007 & 0.003 \\
\hline & $(0.019)$ & $(0.009)$ & $(0.001)$ \\
\hline \multirow[t]{2}{*}{$\alpha_{5}$} & 0.012 & -0.001 & 0.002 \\
\hline & $(0.016)$ & $(0.007)$ & $(0.002)$ \\
\hline \multirow[t]{2}{*}{$\alpha_{6}$} & 0.004 & 0.002 & 0.001 \\
\hline & $(0.013)$ & $(0.006)$ & $(0.001)$ \\
\hline \multirow[t]{2}{*}{$\alpha_{7}$} & 0.012 & 0.010 & 0.004 \\
\hline & $(0.019)$ & $(0.010)$ & $(0.002)$ \\
\hline \multirow[t]{2}{*}{$\beta_{1}$} & -0.032 & -0.010 & -0.003 \\
\hline & $(0.019)$ & $(0.008)$ & $(0.001)$ \\
\hline \multirow[t]{2}{*}{$\beta_{2}$} & -0.017 & -0.014 & -0.006 \\
\hline & $(0.016)$ & $(0.009)$ & $(0.002)$ \\
\hline \multirow[t]{2}{*}{$\beta_{3}$} & -0.038 & -0.009 & 0.001 \\
\hline & $(0.018)$ & $(0.008)$ & $(0.002)$ \\
\hline \multirow[t]{2}{*}{$\beta_{4}$} & -0.028 & -0.012 & -0.006 \\
\hline & $(0.018)$ & $(0.007)$ & $(0.001)$ \\
\hline \multirow[t]{2}{*}{$\beta_{5}$} & -0.034 & -0.008 & -0.001 \\
\hline & $(0.018)$ & $(0.008)$ & $(0.002)$ \\
\hline \multirow[t]{2}{*}{$\beta_{6}$} & -0.016 & -0.010 & 0.000 \\
\hline & $(0.016)$ & $(0.009)$ & $(0.002)$ \\
\hline \multirow[t]{2}{*}{$\beta_{7}$} & -0.019 & -0.011 & 0.003 \\
\hline & $(0.017)$ & $(0.008)$ & $(0.001)$ \\
\hline \multirow[t]{2}{*}{$\lambda_{1}$} & 0.139 & 0.052 & 0.002 \\
\hline & $(2.096)$ & $(0.920)$ & $(0.166)$ \\
\hline \multirow[t]{2}{*}{$\lambda_{2}$} & 0.036 & 0.014 & 0.016 \\
\hline & (1.997) & $(0.849)$ & $(0.177)$ \\
\hline \multirow[t]{2}{*}{$\lambda_{3}$} & 0.188 & 0.012 & 0.013 \\
\hline & $(1.194)$ & $(0.581)$ & $(0.096)$ \\
\hline \multirow[t]{2}{*}{$\lambda_{4}$} & 0.170 & 0.046 & 0.024 \\
\hline & $(1.110)$ & $(0.522)$ & $(0.090)$ \\
\hline \multirow[t]{2}{*}{$\lambda_{5}$} & 0.177 & 0.083 & -0.024 \\
\hline & $(1.269)$ & $(0.662)$ & $(0.120)$ \\
\hline \multirow[t]{2}{*}{$\lambda_{6}$} & 0.069 & 0.044 & -0.001 \\
\hline & (1.048) & $(0.594)$ & $(0.108)$ \\
\hline \multirow[t]{2}{*}{$\lambda_{7}$} & 0.047 & 0.045 & -0.046 \\
\hline & (1.431) & $(0.639)$ & $(0.121)$ \\
\hline
\end{tabular}


tion of period $S=7$ which is an expected result since the series corresponds to daily data. The periodogram provides high peak at frequency 0.14 , which corresponds to the period $=1 / 0.14=7$. The AR order identification per season $\nu=1, \ldots, 7$ is identified by finding the lowest lag for which the sample periodic partial autocorrelation (PePACF) function cuts off ([36]). Tables 3 and 4 present the sample periodic autocorrelation (PeACF) and PePACF functions. In these tables, the values in bold are the sample ACFs that exceeded the confidence intervals given below. The approximate limits of the confidence intervals used in $\mathrm{ACF}$ and PACF tables were constructed for a significance level of $5 \%$. This preliminary model identification step reinforces that a periodic INAR model could be adequate to capture the dynamic of the data.
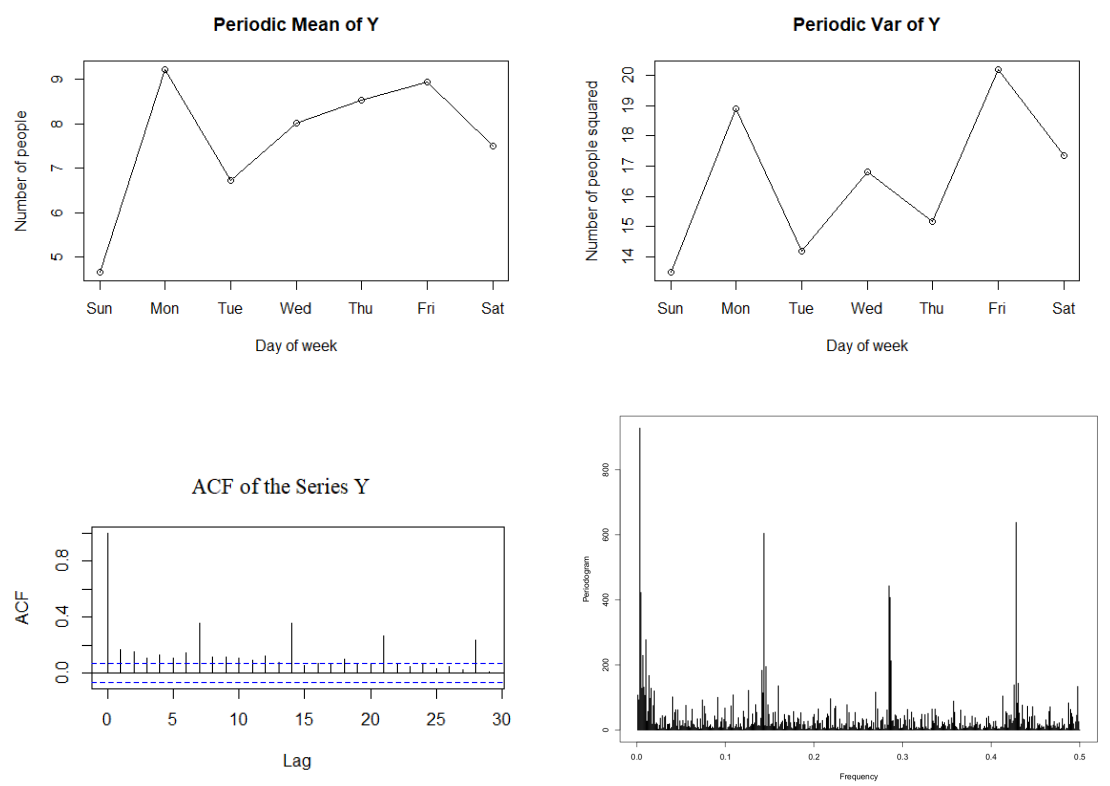

Figure 2: The periodic mean and periodic variance over the seasons $\nu=1, \ldots, 7$, the sample $\mathrm{ACF}$ and the periodogram of $\left\{Y_{t}\right\}$.

Based on the above discussion, the PINAR $\left(1,1_{7}\right)$ Poisson model was used to fit the data. For comparison purpose, three periodic models for count time series were considered. Namely, the standard PINAR(1) Poisson model, the 
Table 3: Periodic ACF of the real data set.

\begin{tabular}{c|rrrrrrrrrrr}
\hline & $h=1$ & $h=2$ & $h=3$ & $h=4$ & $h=5$ & $h=6$ & $h=7$ & $h=8$ & $h=9$ & $h=10$ \\
\hline$\nu=1$ & 0.01 & $\mathbf{0 . 2 6}$ & $\mathbf{0 . 1 8}$ & $\mathbf{0 . 2 4}$ & $\mathbf{0 . 2 8}$ & 0.11 & 0.15 & 0.02 & 0.07 & $\mathbf{0 . 2 9}$ \\
$\nu=2$ & $\mathbf{0 . 3 8}$ & -0.12 & 0.14 & $\mathbf{0 . 2 3}$ & $\mathbf{0 . 1 8}$ & $\mathbf{0 . 1 9}$ & $\mathbf{0 . 2 9}$ & 0.13 & -0.11 & 0.04 \\
$\nu=3$ & $\mathbf{0 . 3 3}$ & $\mathbf{0 . 3 4}$ & -0.02 & 0.10 & $\mathbf{0 . 2 3}$ & $\mathbf{0 . 3 9}$ & $\mathbf{0 . 4 2}$ & $\mathbf{0 . 1 8}$ & $\mathbf{0 . 3 7}$ & 0.03 \\
$\nu=4$ & $\mathbf{0 . 2 7}$ & 0.05 & $\mathbf{0 . 1 7}$ & 0.10 & 0.16 & $\mathbf{0 . 3 3}$ & $\mathbf{0 . 2 9}$ & $\mathbf{0 . 2 3}$ & 0.14 & 0.14 \\
$\nu=5$ & $\mathbf{0 . 1 8}$ & $\mathbf{0 . 3 6}$ & $\mathbf{0 . 2 3}$ & $\mathbf{0 . 3 1}$ & 0.01 & $\mathbf{0 . 1 8}$ & $\mathbf{0 . 2 9}$ & $\mathbf{0 . 2 2}$ & $\mathbf{0 . 2 5}$ & 0.11 \\
$\nu=6$ & $\mathbf{0 . 2 5}$ & 0.16 & $\mathbf{0 . 2 0}$ & 0.14 & 0.16 & $\mathbf{0 . 1 7}$ & $\mathbf{0 . 1 8}$ & $\mathbf{0 . 3 0}$ & $\mathbf{0 . 2 3}$ & 0.13 \\
$\nu=7$ & $\mathbf{0 . 2 0}$ & 0.10 & -0.03 & -0.05 & $\mathbf{- 0 . 1 8}$ & 0.03 & $\mathbf{0 . 3 0}$ & 0.10 & -0.07 & 0.16 \\
\hline
\end{tabular}

Table 4: Periodic PACF of the real data set.

\begin{tabular}{c|rrrrrrrrrr}
\hline \multicolumn{10}{c}{ Table 4: Periodic PACF of the real data set. } \\
\hline \multicolumn{1}{c}{$h=1$} & $h=2$ & $h=3$ & $h=4$ & $h=5$ & $h=6$ & $h=7$ & $h=8$ & $h=9$ & $h=10$ \\
$\nu=2$ & 0.01 & $\mathbf{0 . 2 6}$ & 0.12 & $\mathbf{0 . 2 0}$ & $\mathbf{0 . 1 8}$ & 0.00 & 0.03 & -0.02 & -0.01 & 0.16 \\
$\nu=3$ & $\mathbf{0 . 3 8}$ & -0.14 & 0.08 & $\mathbf{0 . 1 8}$ & 0.07 & 0.01 & $\mathbf{0 . 2 2}$ & -0.06 & -0.08 & -0.02 \\
$\nu=4$ & $\mathbf{0 . 2 7}$ & $\mathbf{0 . 2 4}$ & 0.00 & -0.00 & 0.15 & $\mathbf{0 . 3 2}$ & $\mathbf{0 . 2 9}$ & 0.01 & $\mathbf{0 . 2 6}$ & 0.04 \\
$\nu=5$ & $\mathbf{0 . 1 8}$ & $\mathbf{0 . 3 3}$ & 0.13 & $\mathbf{0 . 1 8}$ & 0.01 & 0.10 & $\mathbf{0 . 1 7}$ & 0.04 & 0.02 & -0.02 \\
$\nu=6$ & $\mathbf{0 . 2 5}$ & 0.12 & 0.10 & 0.06 & 0.03 & $\mathbf{0 . 1 8}$ & 0.08 & $\mathbf{0 . 1 8}$ & 0.13 & -0.05 \\
$\nu=7$ & $\mathbf{0 . 2 0}$ & 0.05 & -0.07 & -0.11 & $\mathbf{- 0 . 2 1}$ & 0.08 & $\mathbf{0 . 2 6}$ & 0.03 & -0.13 & $\mathbf{0 . 2 1}$ \\
\hline
\end{tabular}


$\operatorname{PINARMA}_{S}(p, q)$ Poisson model discussed in [29, and the periodic integervalued generalized autoregressive conditional heteroskedastic, $\operatorname{PINGARCH}_{S}(1,1)$ model introduced in [37. For the $\operatorname{PINAR}\left(1,1_{7}\right)$ and $\operatorname{PINARMA}_{7}(7,0)$ models, the parameters were estimated using the CQML estimation method. To estimate the parameters of the PINAR(1) and $\operatorname{PINGARCH}_{S}(1,1)$ models, the conditional maximum likelihood estimation was used since this method is the one suggested by their respective authors. The Akaike information criterion (AIC) and the Bayesian information criterion (BIC) were calculated for the four models. For the PINAR(1, $\left.1_{7}\right), \operatorname{PINAR}(1), \operatorname{PINARMA}_{7}(7,0)$ and $\operatorname{PINGARCH}_{7}(1,1)$ models, the pair (AIC, BIC) were (7812.33, 2363.47), (7906.29, 2378.19),(7889.95, $2552.59)$ and $(8385.84,2936.99)$, respectively. Based on these quantities, the best model to fit the data is the $\operatorname{PINAR}\left(1,1_{7}\right)$. In addition, the residuals of the PINAR(1), $\operatorname{PINARMA}_{7}(7,0)$ and $\operatorname{PINGARCH}_{7}(1,1)$ models were not accurate, which corroborates the conclusion drawn from the AIC and BIC criteria.

The estimates of the parameters of the $\operatorname{PINAR}\left(1,1_{7}\right)$ model are displayed in Table 5. The standard errors (in parenthesis) were calculated using the inverse of the corresponding Hessian matrix. Figure 3 displays the $95 \%$ confidence intervals of the estimates using the asymptotic normal theory discussed in Theorem 1 The residuals of the model were computed by

$$
r_{t}=y_{t}-\widehat{y}_{t}=y_{t}-\widehat{\alpha}_{\nu} y_{t-1}-\widehat{\beta}_{\nu} y_{t-7}-\widehat{\lambda}_{\nu}
$$

where $t=7 k+\nu, k=1, \ldots, 118$ and $\nu=1, \ldots, 7$. Tables 6 and 7 display the values of PeACF and PePACF functions of the residuals, respectively. We see from these tables that the periodic correlations at lags 1 and 7 were removed and no systematic patterns are observed. The fitted model seems to well capture the main dynamics of the data.

Therefore, it is expected that the estimated model can be useful to provide reliable forecasts. For example, Figure 4 shows the one-step ahead forecasts for the last week of the data. The model to run the forecast was fitted without these observations and presented similar estimates to results displayed in Table 5 . 
Table 5: Application of PINAR $\left(1,1_{7}\right)$ model to the real data. The parameters were estimated by CQML method. The standard errors of the estimates are inside parenthesis.

\begin{tabular}{|c|c|c|c|c|c|c|c|}
\hline Fitted model & $\nu=1$ & $\nu=2$ & $\nu=3$ & $\nu=4$ & $\nu=5$ & $\nu=6$ & $\nu=7$ \\
\hline \multicolumn{8}{|c|}{$\operatorname{PINAR}\left(1,1_{7}\right)$-Poisson Innovation: } \\
\hline$\alpha_{\nu}$ & $0.095(0.039)$ & $0.012(0.074)$ & $0.209(0.045)$ & $0.211(0.061)$ & $0.133(0.060)$ & $0.083(0.056)$ & $0.126(0.045)$ \\
\hline$\beta_{\nu}$ & $0.192(0.047)$ & $0.108(0.054)$ & $0.217(0.055)$ & $0.280(0.056)$ & $0.150(0.061)$ & $0.169(0.053)$ & $0.097(0.051)$ \\
\hline$\lambda_{\nu}$ & $3.031(0.360)$ & $8.209(0.654)$ & $3.364(0.551)$ & $4.361(0.562)$ & $6.182(0.616)$ & $6.739(0.640)$ & $5.649(0.562)$ \\
\hline
\end{tabular}

Table 6: Periodic ACF of residuals of the $\operatorname{PINAR}\left(1,1_{7}\right)$ model.

\begin{tabular}{r|rrrrrrrrrr}
\hline & $h=1$ & $h=2$ & $h=3$ & $h=4$ & $h=5$ & $h=6$ & $h=7$ & $h=8$ & $h=9$ & $h=10$ \\
\hline$\nu=1$ & -0.02 & 0.11 & -0.11 & -0.02 & -0.16 & 0.02 & -0.06 & 0.06 & $\mathbf{- 0 . 2 0}$ & 0.15 \\
$\nu=2$ & -0.04 & $\mathbf{0 . 2 1}$ & 0.04 & 0.13 & 0.13 & 0.04 & -0.04 & 0.02 & -0.05 & $\mathbf{0 . 2 1}$ \\
$\nu=3$ & 0.00 & -0.09 & -0.00 & 0.15 & 0.06 & -0.03 & 0.01 & 0.01 & -0.03 & 0.01 \\
$\nu=4$ & -0.07 & 0.13 & 0.01 & -0.05 & 0.01 & $\mathbf{0 . 2 6}$ & 0.05 & -0.13 & $\mathbf{0 . 2 6}$ & 0.05 \\
$\nu=5$ & -0.02 & -0.10 & 0.05 & 0.08 & 0.04 & $\mathbf{0 . 2 7}$ & -0.06 & 0.07 & 0.11 & 0.04 \\
$\nu=6$ & -0.09 & $\mathbf{0 . 2 3}$ & 0.08 & $\mathbf{0 . 2 7}$ & -0.04 & 0.11 & -0.07 & 0.11 & 0.08 & 0.03 \\
$\nu=7$ & -0.07 & 0.05 & 0.09 & 0.04 & 0.03 & 0.16 & -0.07 & $\mathbf{0 . 2 1}$ & 0.13 & 0.02 \\
\hline
\end{tabular}

Table 7: Periodic PACF of residuals of the PINAR $\left(1,1_{7}\right)$ model.

\begin{tabular}{r|rrrrrrrrrr}
\hline & $h=1$ & $h=2$ & $h=3$ & $h=4$ & $h=5$ & $h=6$ & $h=7$ & $h=8$ & $h=9$ & $h=10$ \\
\hline$\nu=1$ & -0.02 & 0.11 & -0.10 & -0.04 & $-\mathbf{0 . 1 9}$ & -0.00 & -0.06 & 0.05 & -0.13 & 0.14 \\
$\nu=2$ & -0.04 & $\mathbf{0 . 2 1}$ & 0.06 & 0.13 & 0.10 & 0.05 & -0.08 & -0.02 & -0.03 & 0.15 \\
$\nu=3$ & 0.00 & -0.09 & -0.00 & 0.17 & 0.07 & -0.07 & -0.02 & -0.04 & -0.04 & -0.01 \\
$\nu=4$ & -0.07 & 0.13 & 0.01 & -0.08 & 0.00 & $\mathbf{0 . 2 5}$ & 0.04 & -0.10 & $\mathbf{0 . 2 7}$ & 0.05 \\
$\nu=5$ & -0.02 & -0.10 & 0.05 & 0.07 & 0.03 & $\mathbf{0 . 2 8}$ & -0.03 & -0.01 & 0.09 & -0.04 \\
$\nu=6$ & -0.09 & $\mathbf{0 . 2 3}$ & 0.09 & $\mathbf{0 . 2 6}$ & -0.02 & 0.08 & -0.07 & 0.01 & 0.06 & 0.07 \\
$\nu=7$ & -0.07 & 0.04 & 0.11 & 0.06 & 0.04 & 0.16 & -0.07 & 0.17 & 0.17 & -0.01 \\
\hline
\end{tabular}



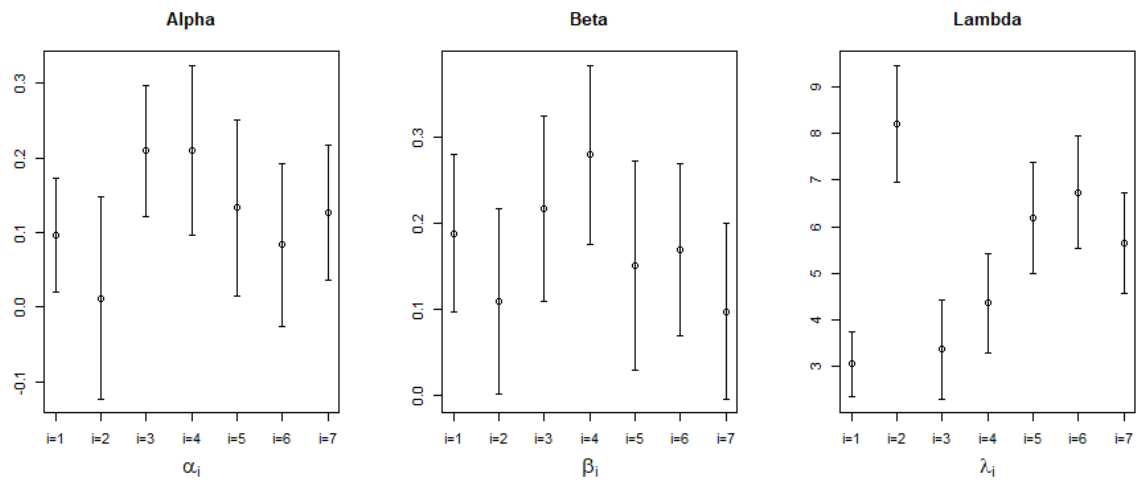

Figure 3: 95\% confidence intervals of the estimates.

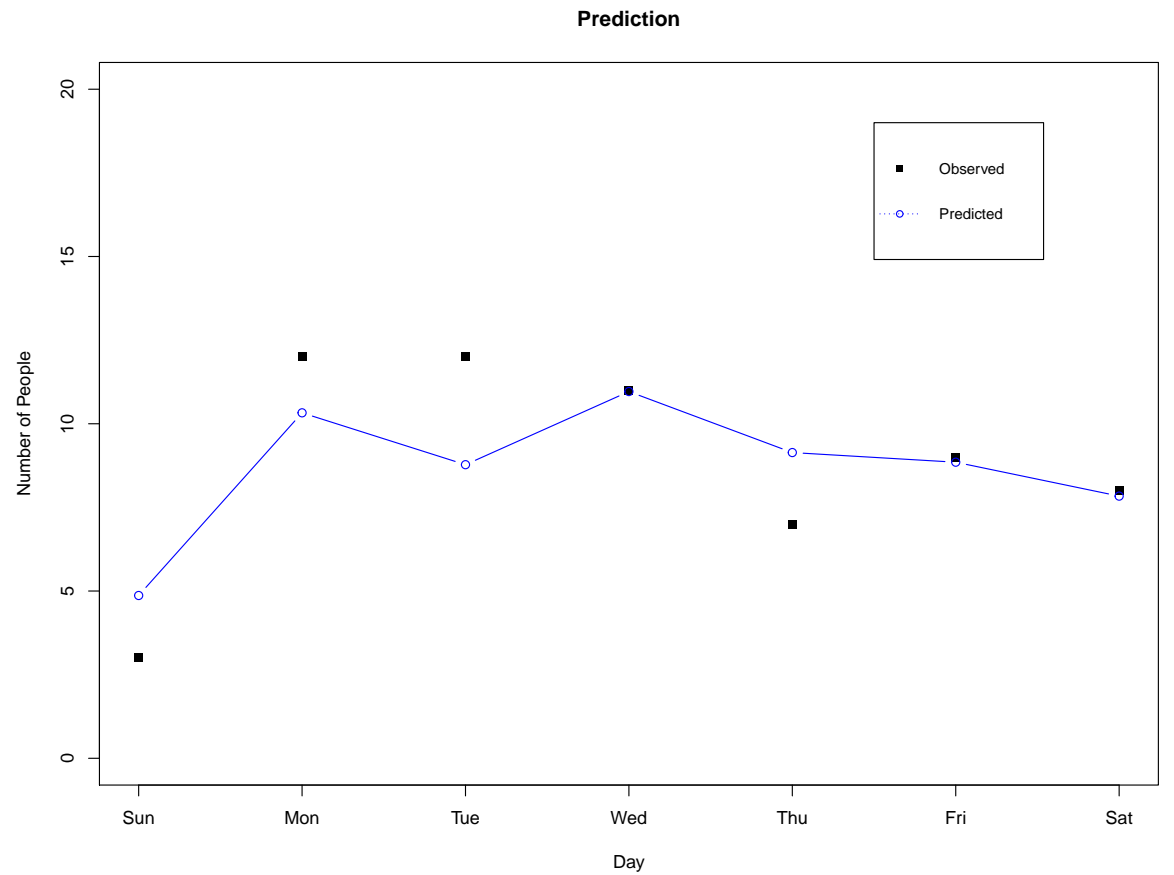

Figure 4: One-step ahead forecasts 


\section{Conclusion}

The PINAR $\left(1,1_{S}\right)$ model with Poisson innovations was introduced and its main properties were established. The CQML estimation method was proposed and a simulation study was carried out to investigate its finite sample properties. The CQML method presented good performances in simulations.

For the very small sample size $n=50$, the estimates of the parameter $\lambda$ were not so much accurate. Therefore, we have also considered an empirical simulation study using the methods proposed in [22] and [29], which make use of the conditional Poisson distribution. Our simulations indicated that the methods performed quite similarly which corroborates the use of the proposed estimation method even when dealing with a time series with small sample size in a real application. These results are available upon request.

The time series of counts of the daily number of people who received antibiotics for the treatment of respiratory problems from the public health care system in the emergency service of the region of Vitória-ES (Brazil) was the main motivation for introducing the $\operatorname{PINAR}\left(1,1_{S}\right)$ model. The $\operatorname{PINAR}\left(1,1_{7}\right)$ model was able to capture the main dynamic of this real data.

The PINAR $\left(1,1_{S}\right)$ model can be extended to more complicated periodic count time series and can be an interesting tool in practical studies, for example, to better understand the behavior dynamic of drug dispensing over time.

\section{Acknowledgments}

The results in this paper are part of the $\mathrm{PhD}$ thesis of the first author in the PPGEA-UFES and CentraleSupélec under supervision of the second and third authors. The first author gratefully acknowledge partial financial support from SmartCities \& SmartGrids for Sustainable Development Project of the Erasmus Mundus Program. Part of this paper was revised when Paulo Prezotti, Valdério Reisen, Márton Ispány and Milena Machado were visiting CentraleSupélec in July 2019. These authors are indebted to CentraleSupélec and Université ParisSaclay for their financial supports. This research was also partially supported 
by the iCODE Institute, research project of the IDEX Paris-Saclay, and by the Hadamard Mathematics LabEx (LMH) through the grant number ANR-11LABX-0056-LMH in the Programme des Investissements d'Avenir. The work of Márton Ispány is also supported by the EFOP-3.6.1-16-2016-00022 project. The project is co-financed by the European Union and the European Social Fund. V. A. Reisen also thanks CNPq and FAPES-ES, Brazil.

The authors express the most sincere gratitude for the valuable comments of the anonymous referees. Their suggestions allow us to substantially improve the quality of the paper.

\section{References}

[1] World Health Organization and others, How to investigate drug use in health facilities: selected drug use indicators, Tech. rep., Geneva: World Health Organization (1993).

[2] R. McDowell, K. Bennett, F. Moriarty, S. Clarke, M. Barry, T. Fahey, An evaluation of prescribing trends and patterns of claims within the Preferred Drugs Initiative in Ireland (2011-2016): an interrupted time-series study, BMJ Open 8 (4) (2018) e019315.

[3] D. Caillaud, M. Cheriaux, S. Martin, C. Ségala, N. Dupuy, B. Evrard, M. Thibaudon, Short-term effect of outdoor mold spore exposure on prescribed allergy medication sales in Central France, Clinical \& Experimental Allergy 48 (7) (2018) 837-845.

[4] A. Oudin, L. Bråbäck, D. Oudin Åström, B. Forsberg, Air pollution and dispensed medications for asthma, and possible effect modifiers related to mental health and socio-economy: A longitudinal cohort study of swedish children and adolescents, International Journal of Environmental Research and Public Health 14 (11) (2017) 1392.

[5] I. Youngster, J. Avorn, V. Belleudi, A. Cantarutti, J. Díez-Domingo, U. Kirchmayer, B.-J. Park, S. Peiró, G. Sanfélix-Gimeno, H. Schröder, 
et al., Antibiotic use in children-a cross-national analysis of 6 countries, The Journal of Pediatrics 182 (2017) 239-244.

[6] J. Holstiege, E. Garbe, Systemic antibiotic use among children and adolescents in Germany: a population-based study, European Journal of Pediatrics 172 (6) (2013) 787-795.

[7] E. McKenzie, Some simple models for discrete variate time series, J. Amer. Water. Res. Assoc. 21 (1985) 645-650.

[8] M. Al-Osh, A. A. Alzaid, First-order integer-valued autoregressive (INAR(1)) process, Journal of Time Series Analysis 8 (3) (1987) 261-275.

[9] L. S. C. Piancastelli, W. Barreto-Souza, Inferential aspects of the zeroinflated Poisson INAR(1) process, Applied Mathematical Modelling 74 (2019) 457-468.

[10] X. Qi, Q. Li, F. Zhu, Modeling time series of count with excess zeros and ones based on INAR(1) model with zero-and-one inflated Poisson innovations, Journal of Computational and Applied Mathematics 346 (2019) $572-590$.

[11] L. Qian, Q. Li, F. Zhu, Modelling heavy-tailedness in count time series, Applied Mathematical Modelling 82 (2020) 766-784.

[12] F. Steutel, K. Van Harn, Discrete analogues of self-decomposability and stability, The Annals of Probability 7 (5) (1979) 893-899.

[13] M. G. Scotto, C. H. Weiss, S. Gouveia, Thinning-based models in the analysis of integer-valued time series: a review, Statistical Modelling 15 (6) (2015) 590-618.

[14] A. Latour, The multivariate GINAR $(p)$ process, Advances in Applied Probability 29 (1) (1997) 228-248. 
[15] A. A. Alzaid, M. Al-Osh, An integer-valued $p$ th-order autoregressive structure (INAR $(p))$ process, Journal of Applied Probability 27 (2) (1990) 314324.

[16] J.-G. Du, Y. Li, The integer-valued autoregressive (INAR $(p)$ ) model, Journal of Time Series Analysis 12 (2) (1991) 129-142.

[17] E. G. Gladyshev, Periodically correlated random sequences, Sov. Math. 2 (1961) 385-388.

[18] W. A. Gardner, A. Napolitano, L. Paura, Cyclostationarity: Half a century of research, Signal Processing 86 (4) (2006) 639-697.

[19] I. Basawa, R. Lund, Large sample properties of parameter estimates for periodic ARMA models, Journal of Time Series Analysis 22 (6) (2001) 651-663.

[20] A. J. Q. Sarnaglia, V. A. Reisen, C. Lévy-Leduc, Robust estimation of periodic autoregressive processes in the presence of additive outliers, Journal of Multivariate Analysis 101 (9) (2010) 2168-2183.

[21] C. C. Solci, V. A. Reisen, A. J. Q. Sarnaglia, P. Bondon, Empirical study of robust estimation methods for PAR models with application to the air quality area, Communications in Statistics - Theory and Methods (2019) $1-17$.

[22] M. Monteiro, M. G. Scotto, I. Pereira, Integer-valued autoregressive processes with periodic structure, Journal of Statistical Planning and Inference 140 (6) (2010) 1529-1541.

[23] M. Sadoun, M. Bentarzi, Efficient estimation in periodic INAR(1) model: parametric case, Communications in Statistics-Simulation and Computation (2019) 1-21.

[24] D. Moriña, P. Puig, J. Ríos, A. Vilella, A. Trilla, A statistical model for hospital admissions caused by seasonal diseases, Statistics in Medicine 30 (26) (2011) 3125-3136. 
[25] M. Bourguignon, K. L. P. Vasconcellos, V. A. Reisen, M. Ispány, A Poisson INAR(1) process with a seasonal structure, Journal of Statistical Computation and Simulation 86 (2) (2016) 373-387.

[26] A. Buteikis, R. Leipus, An integer-valued autoregressive process for seasonality, Journal of Statistical Computation and Simulation 90 (3) (2020) 391-411.

[27] Z. Liu, Q. Li, F. Zhu, Random environment binomial thinning integervalued autoregressive process with Poisson or geometric marginal, Brazilian Journal of Probability and Statistics 34 (2) (2020) 251-272.

[28] A. S. Nastić, P. N. Laketa, M. M. Ristić, Random environment integervalued autoregressive process, Journal of Time Series Analysis 37 (2) (2016) $267-287$.

[29] M. Bentarzi, N. Aries, QMLE of periodic integer-valued time series models, Communications in Statistics-Simulation and Computation (2020) 1-27.

[30] R. A. Horn, C. R. Johnson, Matrix analysis, 2nd Edition, Cambridge University Press, 2012.

[31] E. Seneta, Non-negative matrices and Markov chains, Springer Science \& Business Media, 2006.

[32] F. A. Graybill, Matrices with applications in statistics, Wadsworth Inc., 1983.

[33] S. X. Chen, J. S. Liu, Statistical applications of the Poisson-binomial and conditional Bernoulli distributions, Statistica Sinica 7 (4) (1997) 875-892.

[34] O. Sheynin, Bortkiewicz' alleged discovery: the law of small numbers, Historia scientiarum, Second series: International Journal of the History of Science Society of Japan 18 (1) (2008) 36-48.

[35] M. Taniguchi, Y. Kakizawa, Asymptotic theory of statistical inference for time series, Springer, New York Berlin Heidelberg, 2000. 
[36] A. I. McLeod, Diagnostic checking of periodic autoregression models with application, Journal of Time Series Analysis 15 (2) (1994) 221-233.

[37] M. Bentarzi, W. Bentarzi, Periodic integer-valued $\operatorname{GARCH}(1,1)$ model, Communications in Statistics-Simulation and Computation 46 (2) (2017) $1167-1188$.

\section{Appendix}

The following lemma contains properties of the binomial thinning operator.

Lemma 2. Let $Y$ and $Z$ be $\mathbb{Z}_{+}$-valued r.v.'s and $\alpha, \beta \in[0,1]$. Let $\mathcal{F} \subset \mathcal{A}$ denote a sub-sigma field and $Y, Z$ be $\mathcal{F}$-measurables. If the counting sequences involved in the thinning operators $\alpha \circ$ and $\beta \circ$ are mutually independent and independent of $\mathcal{F}$, then

$P 1$ Conditionally on $Y, \alpha \circ \beta \circ Y \sim \operatorname{Bin}(Y, \alpha \beta)$, i.e., $\alpha \circ \beta \circ Y \stackrel{d}{=}(\alpha \beta) \circ Y$.

$P$ 2 a) $\mathrm{E}(\alpha \circ \beta \circ Y \mid \mathcal{F})=\alpha \beta Y$,

b) $\operatorname{Var}(\alpha \circ \beta \circ Y \mid \mathcal{F})=\alpha \beta(1-\alpha \beta) Y$,

c) $\mathrm{E}((\alpha \circ+\beta \circ) Y \mid \mathcal{F})=(\alpha+\beta) Y$,

d) $\operatorname{Var}((\alpha \circ+\beta \circ) Y \mid \mathcal{F})=(\alpha(1-\alpha)+\beta(1-\beta)) Y$.

$P$ з а) $\mathrm{E}(\alpha \circ \beta \circ Y)=\alpha \beta \mathrm{E}(Y)$,

b) $\operatorname{Var}(\alpha \circ \beta \circ Y)=\alpha \beta(1-\alpha \beta) \mathrm{E}(Y)+(\alpha \beta)^{2} \operatorname{Var}(Y)$,

c) $\mathrm{E}((\alpha \circ+\beta \circ) Y)=(\alpha+\beta) \mathrm{E}(Y)$,

d) $\operatorname{Var}((\alpha \circ+\beta \circ) Y)=(\alpha(1-\alpha)+\beta(1-\beta)) \mathrm{E}(Y)+(\alpha+\beta)^{2} \operatorname{Var}(Y)$.

$P$ 4 a) $\mathrm{E}(\alpha \circ Y+\beta \circ Z \mid \mathcal{F})=\alpha Y+\beta Z$,

b) $\operatorname{Var}(\alpha \circ Y+\beta \circ Z \mid \mathcal{F})=\alpha(1-\alpha) Y+\beta(1-\beta) Z$,

c) $\mathrm{E}(\alpha \circ Y+\beta \circ Z)=\alpha \mathrm{E}(Y)+\beta \mathrm{E}(Z)$,

d) $\operatorname{Var}(\alpha \circ Y+\beta \circ Z)=\alpha(1-\alpha) \mathrm{E}(Y)+\beta(1-\beta) \mathrm{E}(Z)+\alpha^{2} \operatorname{Var}(Y)+$ $2 \alpha \beta \operatorname{Cov}(Y, Z)+\beta^{2} \operatorname{Var}(Z)$. 
Proof of Lemma 2. (P1) Conditionally on $\mathcal{F}, \alpha \circ Y$ defined in (1), follows a $\operatorname{Bin}(Y, \alpha)$, for any $\alpha \in[0,1]$. Therefore, $\mathrm{P} 1$ is easily obtained. (P2) The first two statements follow from (P1) and the independence of the thinning operators. The last two statements are a direct consequence of the definition of the thinning operator. (P3) comes from (P2) by the law of total variance. Finally, (P4) can be proved as (P2) and (P3).

Proof of Proposition 1. By (2) and bilinearity of covariance, we have for all $h>0$,

$$
\operatorname{Cov}\left(Y_{t}, Y_{t-h}\right)=\operatorname{Cov}\left(\alpha_{i} \circ Y_{t-1}, Y_{t-h}\right)+\operatorname{Cov}\left(\beta_{i} \circ Y_{t-S}, Y_{t-h}\right)+\operatorname{Cov}\left(\epsilon_{t}, Y_{t-h}\right)
$$

Since $\epsilon_{t}$ is independent of $Y_{t-h}, \operatorname{Cov}\left(\epsilon_{t}, Y_{t-h}\right)=0$. Using the law of total covariance, we get

$\operatorname{Cov}\left(\alpha_{i} \circ Y_{t-1}, Y_{t-h}\right)=\mathrm{E}\left(\operatorname{Cov}\left(\alpha_{i} \circ Y_{t-1}, Y_{t-h} \mid \mathcal{F}_{t-1}\right)\right)+\operatorname{Cov}\left(\mathrm{E}\left(\alpha_{i} \circ Y_{t-1} \mid \mathcal{F}_{t-1}\right), \mathrm{E}\left(Y_{t-h} \mid \mathcal{F}_{t-1}\right)\right)$.

Since

$\operatorname{Cov}\left(\alpha_{i} \circ y_{t-1}, y_{t-h}\right)=\mathrm{E}\left(\left(\alpha_{i} \circ y_{t-1}\right) y_{t-h}\right)-\mathrm{E}\left(\alpha_{i} \circ y_{t-1}\right) \mathrm{E}\left(y_{t-h}\right)=y_{t-h}\left(\alpha_{i} y_{t-1}\right)-\left(\alpha_{i} y_{t-1}\right) y_{t-h}=0$, the first term in 13$)$ equals zero. Since $\mathrm{E}\left(\alpha_{i} \circ Y_{t-1} \mid \mathcal{F}_{t-1}\right)=\alpha_{i} Y_{t-1}$ and $\mathrm{E}\left(Y_{t-h} \mid \mathcal{F}_{t-1}\right)=Y_{t-h}$, we deduce from (13) that $\operatorname{Cov}\left(\alpha_{i} \circ Y_{t-1}, Y_{t-h}\right)=\alpha_{i} \operatorname{Cov}\left(Y_{t-1}, Y_{t-h}\right)$.

Similarly, $\operatorname{Cov}\left(\beta_{i} \circ Y_{t-S}, Y_{t-h}\right)=\beta_{i} \operatorname{Cov}\left(Y_{t-S}, Y_{t-h}\right)$ and the statement follows.

Proof of Proposition 2,

$$
\begin{gathered}
P\left(Y_{t}=y_{t}, \ldots, Y_{S+1}=y_{S+1} \mid Y_{S}=y_{S}, \ldots, Y_{1}=y_{1}\right)= \\
\frac{P\left(Y_{t}=y_{t}, \ldots, Y_{1}=y_{1}\right)}{P\left(Y_{t-1}=y_{t-1}, \ldots, Y_{1}=y_{1}\right)} \cdot \frac{P\left(Y_{t-1}=y_{t-1}, \ldots, Y_{1}=y_{1}\right)}{P\left(Y_{S}=y_{S}, \ldots, Y_{1}=y_{1}\right)}= \\
P\left(Y_{t}=y_{t} \mid Y_{t-1}=y_{t-1}, \ldots, Y_{1}=y_{1}\right) \times \\
P\left(Y_{t-1}=y_{t-1}, \ldots, Y_{S+1}=y_{S+1} \mid Y_{S}=y_{S}, \ldots, Y_{1}=y_{1}\right)= \\
p_{\nu}\left(y_{t} \mid y_{t-1}, y_{t-S}\right) P\left(Y_{t-1}=y_{t-1}, \ldots, Y_{S+1}=y_{S+1} \mid Y_{S}=y_{S}, \ldots, Y_{1}=y_{1}\right),
\end{gathered}
$$

where $t=k S+\nu, t>S$ and $y_{1}, \ldots, y_{t} \in \mathbb{Z}_{+}$. Thus, by induction, if $T=n S$ where $n \in \mathbb{N}$, the conditional probability can be calculated by (7). 
Proof of Theorem 1. The proof is based on the conditions in [35, Theorem 3.2.26]. Let $\left\{Y_{t}\right\}_{t \in \mathbb{Z}}$ be a $\operatorname{PINAR}\left(1,1_{S}\right)$ process and $\left\{\boldsymbol{Y}_{k}\right\}_{k \in \mathbb{Z}}$ defined by (4) where $A, B$ satisfy Assumption 1. By Lemma 11, for each $\nu,\left\{Y_{k S+\nu}\right\}_{k \in \mathbb{Z}}$ is a strictly stationary ergodic process. Let $Y^{*}=\left\{Y_{1}, \ldots, Y_{T}\right\}$ be a sample of $\left\{Y_{t}\right\}_{k \in \mathbb{Z}}$. Functions $m_{k, \nu}\left(\vartheta_{\nu}\right)$ and $f_{k, \nu}\left(\vartheta_{\nu}\right)$ defined by (9) and (10), respectively, are almost surely three times continuously differentiable in the open set $\Theta$ which contains $\boldsymbol{\vartheta}_{0}$. Then, function $\phi_{k, \nu}\left(\vartheta_{\nu}\right)$ defined by (11) is also almost surely three times continuously differentiable in $\Theta$. Theorem 1 is proved if $\phi_{k, \nu}\left(\vartheta_{\nu}\right)$ satisfies the following conditions:

C1. For $1 \leq i, j \leq 3$,

$$
\mathrm{E}\left\{\left|\frac{\partial}{\partial\left(\vartheta_{\nu}\right)_{i}} \phi_{k, \nu}\left(\vartheta_{\nu}^{0}\right)\right|\right\}<\infty \text { and } \mathrm{E}\left\{\left|\frac{\partial^{2}}{\partial\left(\vartheta_{\nu}\right)_{i} \partial\left(\vartheta_{\nu}\right)_{j}} \phi_{k, \nu}\left(\vartheta_{\nu}^{0}\right)\right|\right\}<\infty \text {, }
$$

where $\left(\vartheta_{\nu}\right)_{i}$ is the $i$ th element of the vector $\left(\vartheta_{\nu}\right)$, that is, $\left(\vartheta_{\nu}\right)_{1}=\alpha_{\nu},\left(\vartheta_{\nu}\right)_{2}=\beta_{\nu}$ and $\left(\vartheta_{\nu}\right)_{3}=\lambda_{\nu}$.

C2. The functions

$$
f_{k, \nu}\left(\vartheta_{\nu}^{0}\right)^{-1 / 2} \frac{\partial}{\partial\left(\vartheta_{\nu}\right)_{i}} m_{k, \nu}\left(\vartheta_{\nu}^{0}\right),
$$

for $i=1,2,3$, are linearly independent.

C3. For enery $\boldsymbol{\vartheta} \in \Theta$, there exists a function $H_{t}^{i j l}\left(Y_{1}, \ldots, Y_{T}\right.$ such that

$$
\left|\frac{\partial^{3}}{\partial\left(\vartheta_{\nu}\right)_{i} \partial\left(\vartheta_{\nu}\right)_{j} \partial\left(\vartheta_{\nu}\right)_{l}} \phi_{k, \nu}\left(\vartheta_{\nu}\right)\right| \leq H_{t}^{i j l}
$$

with $\mathrm{E}\left(H_{t}^{i j l}\right)<\infty$ for $i, j, l=1,2,3$.

$\mathrm{C} 4$.

$$
V=\mathrm{E}\left\{\frac{\partial}{\partial\left(\vartheta_{\nu}\right)} \phi_{k, \nu}\left(\vartheta_{\nu}^{0}\right) \frac{\partial}{\partial\left(\vartheta_{\nu}\right)^{\top}} \phi_{k, \nu}\left(\vartheta_{\nu}^{0}\right)\right\}<\infty .
$$

In the following, we check $\mathrm{C} 1, \ldots, \mathrm{C} 4$ for the $\operatorname{PINAR}\left(1,1_{S}\right)$ model.

Let $g_{k, \nu}\left(\vartheta_{\nu}\right)=Y_{t}-m_{k, \nu}\left(\vartheta_{\nu}\right)$. We have

$$
\begin{aligned}
& \frac{\partial}{\partial\left(\vartheta_{\nu}\right)_{i}} \phi_{k, \nu}\left(\vartheta_{\nu}\right)=\frac{1}{f_{k, \nu}\left(\vartheta_{\nu}\right)} \frac{\partial}{\partial\left(\vartheta_{\nu}\right)_{i}} f_{k, \nu}\left(\vartheta_{\nu}\right)+\frac{2 g_{k, \nu}\left(\vartheta_{\nu}\right)}{f_{k, \nu}\left(\vartheta_{\nu}\right)} \frac{\partial}{\partial\left(\vartheta_{\nu}\right)_{i}} g_{k, \nu}\left(\vartheta_{\nu}\right)- \\
& \frac{g_{k, \nu}^{2}\left(\vartheta_{\nu}\right)}{f_{k, \nu}^{2}\left(\vartheta_{\nu}\right)} \frac{\partial}{\partial\left(\vartheta_{\nu}\right)_{i}} f_{k, \nu}\left(\vartheta_{\nu}\right),
\end{aligned}
$$


and

$$
\begin{aligned}
& \frac{\partial^{2}}{\partial\left(\vartheta_{\nu}\right)_{i}\left(\vartheta_{\nu}\right)_{j}} \phi_{k, \nu}\left(\vartheta_{\nu}\right)=\frac{-1}{f_{k, \nu}^{2}\left(\vartheta_{\nu}\right)} \frac{\partial}{\partial\left(\vartheta_{\nu}\right)_{i}} f_{k, \nu}\left(\vartheta_{\nu}\right) \frac{\partial}{\partial\left(\vartheta_{\nu}\right)_{j}} f_{k, \nu}\left(\vartheta_{\nu}\right)+\frac{1}{f_{k, \nu}\left(\vartheta_{\nu}\right)} \\
& \frac{\partial^{2}}{\partial\left(\vartheta_{\nu}\right)_{i} \partial\left(\vartheta_{\nu}\right)_{j}} f_{k, \nu}\left(\vartheta_{\nu}\right)+2 \frac{\partial}{\partial\left(\vartheta_{\nu}\right)_{i}}\left(\frac{g_{k, \nu}\left(\vartheta_{\nu}\right)}{f_{k, \nu}\left(\vartheta_{\nu}\right)}\right) \frac{\partial}{\partial\left(\vartheta_{\nu}\right)_{j}} g_{k, \nu}\left(\vartheta_{\nu}\right)+\frac{2 g_{k, \nu}\left(\vartheta_{\nu}\right)}{f_{k, \nu}\left(\vartheta_{\nu}\right)} \\
& \frac{\partial^{2}}{\partial\left(\vartheta_{\nu}\right)_{i} \partial\left(\vartheta_{\nu}\right)_{j}} g_{k, \nu}\left(\vartheta_{\nu}\right)-\frac{\partial}{\partial\left(\vartheta_{\nu}\right)_{i}}\left(\frac{g_{k, \nu}^{2}\left(\vartheta_{\nu}\right)}{f_{k, \nu}^{2}\left(\vartheta_{\nu}\right)}\right) \frac{\partial}{\partial\left(\vartheta_{\nu}\right)_{j}} f_{k, \nu}\left(\vartheta_{\nu}\right)- \\
& \frac{g_{k, \nu}^{2}\left(\vartheta_{\nu}\right)}{f_{k, \nu}^{2}\left(\vartheta_{\nu}\right)} \frac{\partial^{2}}{\partial\left(\vartheta_{\nu}\right)_{i} \partial\left(\vartheta_{\nu}\right)_{j}} f_{k, \nu}\left(\vartheta_{\nu}\right) .
\end{aligned}
$$

It follows form 10 that

$$
\frac{1}{f_{k, \nu}\left(\vartheta_{\nu}\right)} \frac{\partial}{\partial\left(\vartheta_{\nu}\right)_{i}} f_{k, \nu}\left(\vartheta_{\nu}\right)= \begin{cases}\frac{\left(1-2 \alpha_{\nu}\right) Y_{t-1}}{f_{k, \nu}\left(\vartheta_{\nu}\right)} & \text { if } i=1 \\ \frac{\left(1-2 \beta_{\nu}\right) Y_{t-S}}{f_{k, \nu}\left(\vartheta_{\nu}\right)} & \text { if } i=2 \\ \frac{1}{f_{k, \nu}\left(\vartheta_{\nu}\right)} & \text { if } i=3\end{cases}
$$

Since $f_{k, \nu}\left(\vartheta_{\nu}\right) \geq \lambda_{\nu}>0,1 / f_{k, \nu}\left(\vartheta_{\nu}\right) \leq 1 / \lambda_{\nu}$, and the absolute value of the first term in the right hand side of 17 is bounded by the integrable $\mathbb{Z}_{+}$-valued r.v.'s, $c Y_{t-1}, c Y_{t-S}$ or $c$, where $c$ is a positive number, this first term satisfies $\mathrm{C} 1$. For simplicity, we write in the following "bounded by $c Y_{t}$ ". Similarly, one can prove that the second and third terms in the right hand side of (17) are bounded by $c Y_{t}^{2}$, and all the terms in the right hand side of 18 are bounded by $c Y_{t}^{3}$. Since $\mathrm{E}\left(Y_{t}^{3}\right)<\infty$, this implies C1.

According to (9), $\frac{\partial}{\partial \vartheta_{\nu}} m_{k, \nu}\left(\vartheta_{\nu}^{0}\right)=\left(Y_{t-1}, Y_{t-S}, 1\right)$. To prove $\mathrm{C} 2$, it is sufficient to show that the r.v.'s $Y_{t-1}, Y_{t-S}$ and 1 are linearly independent. Suppose that $a_{1}, a_{2}, a_{3}$ are real numbers such that $a_{1} Y_{t-1}+a_{2} Y_{t-S}+a_{3}=0$. Then, $\operatorname{Cov}\left(a_{1} Y_{t-1}+a_{2} Y_{t-S}+a_{3}, Y_{t-h}\right)=0, \forall h \in \mathbb{Z}$, i.e, $a_{1} \operatorname{Cov}\left(Y_{t-1}, Y_{t-h}\right)+$ $a_{2} \operatorname{Cov}\left(Y_{t-S}, Y_{t-h}\right)=0$. If $t-1 \equiv(i \bmod S), i=1, \ldots, S$, then we have $a_{1} \gamma_{i}(h-1)+a_{2} \gamma_{i+1}(h-S)=0$. When $i=S, i+1=S+1 \equiv(1 \bmod S)$.

Then we have, $\forall h \in \mathbb{Z}$,

$$
\left\{\begin{array}{l}
a_{1} \gamma_{i}(h-1)+a_{2} \gamma_{i+1}(h-S)=0, \text { for } i=1, \ldots, S-1, \\
a_{1} \gamma_{S}(h-1)+a_{2} \gamma_{1}(h-S)=0 .
\end{array}\right.
$$


The unique solution to the above system is $a_{1}=a_{2}=0$, and then we deduce that $a_{1}=a_{2}=a_{3}=0$.

The proof of $\mathrm{C} 3$ follows from easy but fastidious calculations involving the third derivatives of $\phi_{k, \nu}\left(\vartheta_{\nu}\right)$.

Since each derivative $\frac{\partial}{\partial\left(\vartheta_{\nu}\right)_{i}} \phi_{k, \nu}\left(\vartheta_{\nu}^{0}\right)$ is bounded by $c Y_{t}^{2}$, each term in

$$
\frac{\partial}{\partial\left(\vartheta_{\nu}\right)} \phi_{k, \nu}\left(\vartheta_{\nu}^{0}\right) \frac{\partial}{\partial\left(\vartheta_{\nu}\right)^{\top}} \phi_{k, \nu}\left(\vartheta_{\nu}^{0}\right)
$$

is bounded by $c Y_{t}^{4}$. Since $\mathrm{E}\left(\varepsilon_{t}^{4}\right)<\infty$, we have $\mathrm{E}\left(Y_{t}^{4}\right)<\infty$ and then $V$ is finite.

Full postal address of the corresponding author: Rua jose pinto da silva 65, Apt 804, Mata da Praia, Vitoria, Espirito Santo, Brazil, CEP: 29066-160. 